\title{
Manejo de enfermedad hepática metastásica colorrectal bilateral: Colectomía y hepatectomía multi-etapas: Experiencia inicial*
}

\author{
Drs. FRANCO INNOCENTI C. ${ }^{1,2}$, MISAEL OCARES U..$^{1,2}$, CARMEN SANTANDER A. ${ }^{2}$, \\ ERNESTO HINRICHS O. ${ }^{1,2}$, OSCAR LYNCH G. ${ }^{2,3}$, FELIPE MARTIN Q. ${ }^{2,3}$, \\ CRISTIAN LEDERMANN S. ${ }^{1,2}$, ELIZABETH ROSS S. ${ }^{2,3}$ \\ 1 Departamento de Cirugía, Universidad de Concepción. \\ 2 Clínica Sanatorio Alemán de Concepción. \\ 3 Hospital Clínico Regional de Concepción. \\ Concepción, Chile.
}

\begin{abstract}
Two stage hepatectomy and colectomy among patients with bilateral liver metastases. Report of eight cases

Background: Two stage hepatectomy takes advantage of the compensatory regeneration of the liver after a first non curative hepatectomy, to achieve a curative excision in a second intervention. Aim: To report an initial experience with two stage hepatectomy. Material and Methods: Out of sixty four patients who underwent hepatectomy in our institution in the presented period, eight met criteria for colorectal primary and bilateral liver metastatic disease, which we report. Results: Eight patients were subjected to a right portal ligation. In four, a simultaneous metastasis excision in the left lobe was performed. Four to eight weeks later, five were subjected to a right hepatectomy. One patient, that had a relapse of a previous hepatectomy and colectomy, was explored, ligated and afterwards subjected to surgery plus radiofrequency. The six patients that were subjected to excisions were followed for 3 to 39 months. One patient died due to massive pulmonary relapse at 18 months, two were operated again and subjected to radiofrequency, without evidence of residual disease. Two patients have no evidence of relapse and one had a relapse in a rib. Conclusions: In our series, two stage hepatectomy among patients with colorectal cancer and liver metastasis, but its long term results must be evaluated.
\end{abstract}

Key words: Hepatectomy, liver metastases, colorectal cancer.

\section{Resumen}

Introducción: La hepatectomía en dos etapas utiliza la regeneración compensatoria del hígado tras una primera hepatectomía no curativa para lograr una resección curativa en una segunda intervención. Objetivo: Presentar nuestra experiencia inicial aprovechando esta propiedad, en la estrategia de manejo de pacientes previamente declarados irresecables. Material y Método: Entre Marzo de 2006 y Marzo de 2010, fueron tratados 8 casos. Resultados: De 64 pacientes en los que se realizó hepatectomía, 8 de ellos, con enfermedad metastásica bilateral, confinada al hígado, fueron sometidos a ligadura portal del lado derecho; en la mitad

* Recibido el 10 de Noviembre de 2009 y aceptado para publicación el 29 de Abril de 2010.

Correspondencia: Dr. Franco Innocenti C.

Alto Llacolén 776. Idahue, San Pedro de La Paz, Región del Bío-Bío.

E-mail: f.innocenti@vtr.net 
se realizó resección simultánea de enfermedad del futuro remanente hepático izquierdo. Cuatro a 8 semanas más tarde, cinco fueron sometidos a hepatectomía derecha extendida o hepatectomía derecha (con segmento IV previamente resecado), y otro que era recurrente de previa hepatectomía central más colectomía, fue explorado, ligado y luego sometido a cirugía combinada con radiofrecuencia (RFA). Con seguimiento promedio para el grupo resecado $\mathrm{R} 0(\mathrm{n}=6)$ de 18 meses $(3-39)$, una paciente fallece por recurrencia pulmonar masiva a los 18 meses del diagnóstico, dos se someten a tercera cirugía y RFA con intención curativa, sin evidencia actual de enfermedad, dos no presentan recurrencia y una presenta recurrencia costal, sin evidencia de enfermedad intraabdominal. Otros dos están esperando la hepatectomía post resección del primario y ligadura portal. Conclusión: En nuestro medio, la colectomía y hepatectomía en múltiples etapas en pacientes con enfermedad hepática bilateral metastásica de origen colorrectal es una estrategia factible y razonable para lograr una resección con intención curativa.

Palabras clave: Metástasis hepáticas colorrectales bilaterales, hepatectomía en dos etapas, ligadura vena porta, hiperplasia hepática compensatoria.

\section{Introducción}

Un $80 \%$ de los pacientes con metástasis hepáticas colorrectales (MHCC) son irresecables al momento del diagnóstico. La resección hepática es la única opción terapéutica potencialmente curativa, comparada muy favorablemente con quimioterapia o terapia biológica sin resección ${ }^{1-10}$. Se conocen factores de riesgo y predictores de recurrencia para seleccionar mejor aquellos casos candidatos a cirugía ${ }^{8,10,11}$. Se han desarrollado también estrategias ablativas no resectivas tales como criocirugía, embolización o radiofrecuencia (RFA) sin lograr mejorar resultados de la cirugía ${ }^{5,6,10}$.

El concepto y resultados iniciales de la hepatectomía en 2 etapas fueron desarrollados por Adam ${ }^{1,2}$ en el 2000. Esta estrategia fue propuesta para pacientes con MHCC múltiples bilaterales imposibles de resecar en una sola hepatectomía, aún en combinación con neoadyuvancia y las técnicas anteriormente mencionadas.

Este procedimiento consiste en resecar en un primer tiempo la enfermedad del lado menos afectado, ligadura portal del más comprometido (con primario ya resuelto o en forma simultánea) y realizar en un segundo tiempo la resección potencialmente curativa, basándose en la regeneración compensatoria del futuro hígado remanente.

En reportes se ha visto que proporciona una mejoría significativa en la sobrevida de un grupo de pacientes con metástasis hepáticas colorrectales múltiples bilaterales que de otra manera recibirían sólo un tratamiento paliativo ${ }^{2,5,6}$.

El objetivo de este estudio es presentar nuestra experiencia inicial con pacientes sometidos a cirugía en múltiples etapas.

\section{Material y Método}

En el período entre Marzo de 2006 y Marzo de 2009 , se incluye en la serie a pacientes con enferme- dad metastásica bilateral no resecable por extensión de la enfermedad o por hígado remanente estimado menor al $30 \%$ post resección, con o sin quimioterapia previa. En aquellos pacientes sin enfermedad en futuro hígado remanente pero estimado menor al $30 \%$ se plantea embolización portal percutánea contralateral previa a la cirugía definitiva. En aquellos pacientes con primario obstructivo y con enfermedad hepática bilateral que compromete el futuro hígado remanente, se plantea resección del primario, resección de enfermedad del futuro remanente hepático y ligadura portal contralateral simultáneos. En todos se realiza linfadenectomía con biopsia rápida de linfonodos intercavo aórticos supra e infrarrenales. Se continúa con plan trazado si estos ganglios son negativos. En el caso de no presentar enfermedad de un futuro remanente hepático menor al 30\% del volumen, si es necesaria resección urgente del primario se efectúa ligadura portal contralateral simultánea. Subsecuentemente se realiza hepatectomía con intención curativa del lado ligado. En aquellos pacientes con primario ya resecado, con enfermedad en futuro hígado remanente, se plantea también cirugía o hepatectomía en dos etapas. La indicación de cirugía se plantea cuando lo anterior sea técnicamente factible, de lo contrario se intenta citorreducción preoperatoria con adyuvancia. Los pacientes se estudian endoscópicamente, TAC de tórax, abdomen (con volumetría hepática) y pelvis, y/o resonancia magnética (RNM) y cintigrama óseo. En algunos casos se estudia con Tomografía Computada más Tomografía de Emisión Positrónica (PET $\mathrm{CT})$ si se sospecha diseminación peritoneal u otra a distancia (Ej. dolor abdominal distante o imágenes dudosas). También se evalúa función hepática, antígeno carcinoembrionario (CEA) y clínica, por un equipo multidisciplinario. En todos se controla la respuesta post ligadura portal con TAC previo a segunda etapa de hepatectomía. Son sometidos a seguimiento postoperatorio con evaluación clínica, imagenológica y de laboratorio. 


\section{Resultados}

Se presentan a continuación ocho casos. Seis de ellos corresponden a hepatectomía en dos etapas o a cirugía hepática en dos etapas más la resección del primario. Otros dos fueron sometidos a resección de futuro remanente y ligadura portal derecha y esperan por su hepatectomía de segunda etapa. No hubo candidatos a embolización percutánea en nuestra serie.

\section{Caso 1}

Mujer de 54 años diagnosticada en diciembre de 2007 con tumor de recto y extensa enfermedad bilateral (Figura 1). CEA normal. Recibió radioterapia en el tumor primario y 8 ciclos con capecitabina, oxaliplatino (Esquema XELOX) y bevacizumab. En un primer tiempo se realiza proctocolectomía con resección transesfinteriana, hepatectomía izquierda segmentaria asociada a metastasectomía izquierda

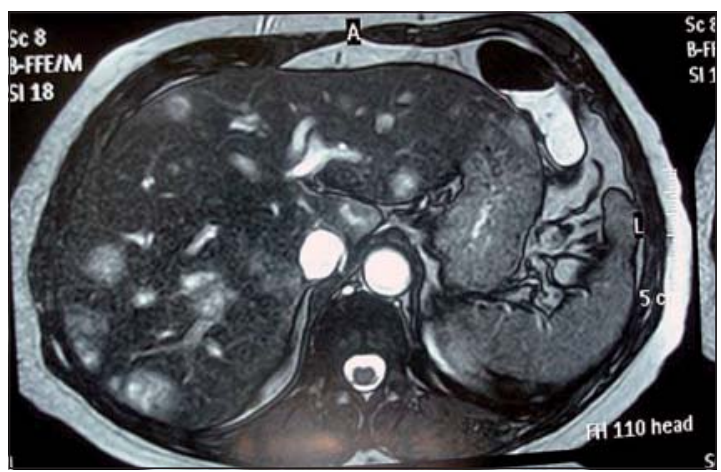

Figura 1. Se aprecian múltiples lesiones metastásicas bilaterales.

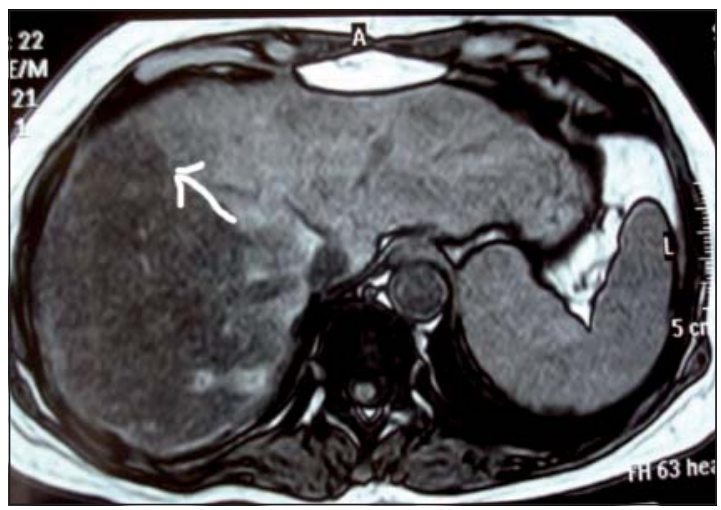

Figura 3. Imagen que evidencia hipoperfusión del hígado derecho post ligadura portal. La flecha blanca indica la interfase entre ambos territorios.
(Figura 2) y ligadura portal derecha. Cursa con una adecuada hipertrofia del hemihígado izquierdo evidenciada por RNM (Figura 3). Tras 8 semanas se realizó una hepatectomía derecha extendida. Evoluciona sin complicaciones postoperatorias y hospitalización de 9 días. La biopsia informa 24 nódulos de metástasis de carcinoma escamoso basaloide moderadamente diferenciado en hígado derecho con márgenes negativos (Figura 4). A los 12 meses de operada, presenta recurrencia pulmonar y hepática, falleciendo 16 meses después de la segunda cirugía y 18 meses post diagnóstico.

\section{Caso 2}

Mujer de 58 años cuya presentación clínica correspondió a un cuadro de pielonefritis aguda asociada a distensión abdominal. Dentro de su estudio, la colonoscopia evidenció una neoplasia subobstructiva de colon izquierdo, con CEA de $444 \mathrm{ng} / \mathrm{ml}$. Imáge-

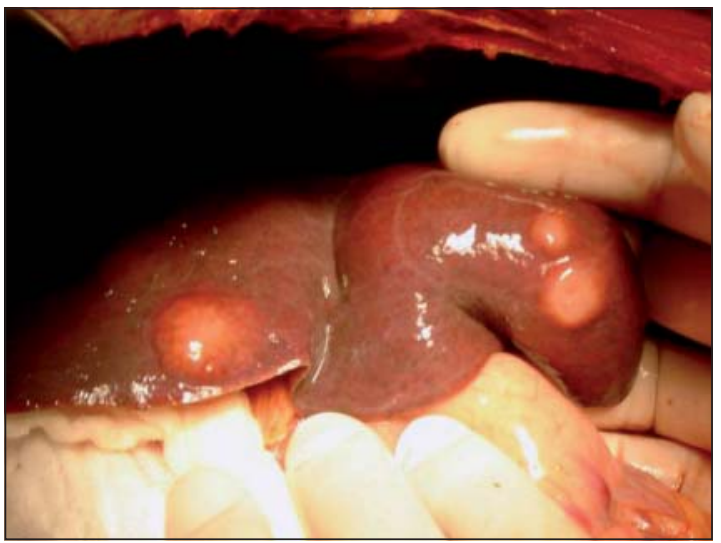

Figura 2. Se observa enfermedad en futuro remanente hepático, segmentos 2 y 3 .

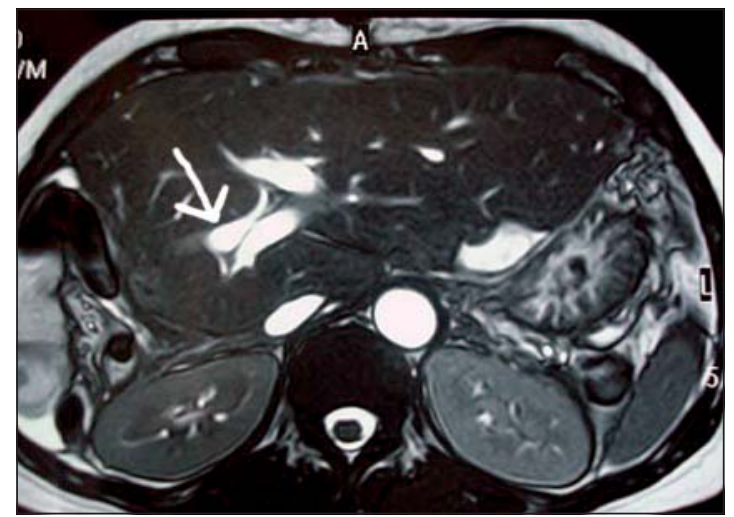

Figura 4. Control imagenológico post hepatectomía derecha. Nótese la gran hipertrofia del hígado izquierdo. La flecha blanca indica el tronco portal izquierdo. 
nes revelan grandes lesiones, una de ellas mayor de $10 \mathrm{~cm}$ con compromiso de vena suprahepática media y porta derecha. No se realiza neoadyuvancia. En un primer tiempo se efectúa hemicolectomía izquierda y ligadura portal derecha evolucionando sin complicaciones. Tras 4 semanas es sometida a hepatectomía derecha extendida, evolucionando sin complicaciones tras una estadía postoperatoria de 12 días. La biopsia informa 14 nódulos metastáticos de adenocarcinoma tubular de origen colónico. Recibe Folfox-Avastín, con un CEA menor a $2 \mathrm{ng} / \mathrm{ml}$ al mes postoperatorio. Presenta recurrencia costal focal manejada con radioterapia a los 14 meses, sin evidencia de recurrencia intraabdominal a RNM y PET CT. Con 24 meses de seguimiento postoperatorio, enfermedad sin signos de progresión.

\section{Caso 3}

Mujer de 34 años, durante estudio colonoscópico por rectorragia en abril de 2008 se encuentra una neoplasia colorrectal. TAC de abdomen muestra nódulos hepáticos bilaterales, con CEA de $1.650 \mathrm{ng} /$ $\mathrm{ml}$. Cursa neoadyuvancia con radioterapia y 6 ciclos de fluoruracilo, oxaliplatino, leucovorina (Esquema FOLFOX) y bevacizumab, presentando respuesta de lesiones hepáticas y caída del CEA a $45 \mathrm{ng} / \mathrm{ml}$. En un primer tiempo se realiza colectomía parcial, segmentectomía IV, metastasectomía del segmento III y ligadura portal derecha, sin complicaciones postoperatorias. La histopatología informa adenocarcinoma tubular moderadamente diferenciado en el primario; necrosis y fibrosis hialina, sin signos de neoplasia en segmentos IV y nódulo del segmento III. A las 6 semanas es sometida a hepatectomía derecha, evolucionando con infección de herida operatoria por S. aureus, permaneciendo hospitalizada 18 días. La biopsia informa 5 nódulos necróticos sin enfermedad

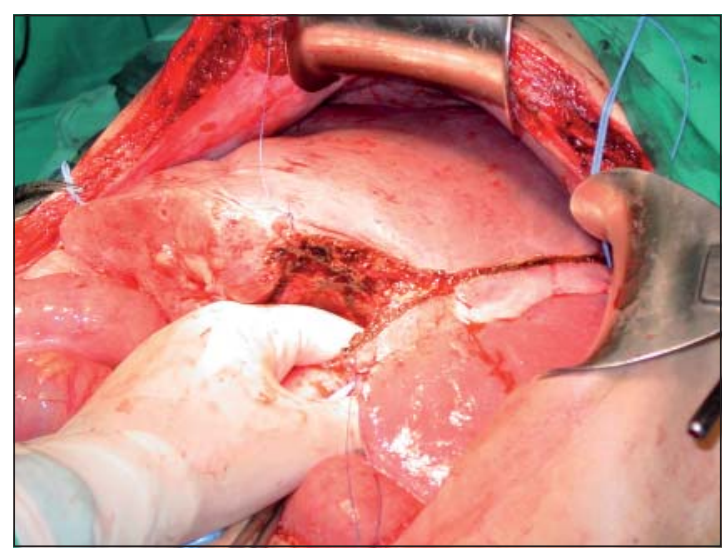

Figura 5. Se inicia la transección para hepatectomía derecha extendida. residual y un nódulo de $0,8 \mathrm{~cm}$ con adenocarcinoma metastático viable. Presenta recurrencia hepática a los 12 meses en hígado remanente, lejos del borde de resección. Recibe quimioterapia fluoruracilo, leucovorina, irinotecán (Esquema FOLFIRI) y bevacizumab. Hay cuatro nódulos hepáticos y ganglios inguinales izquierdos sospechosos al PET CT. A los 16 meses de la segunda etapa de hepatectomía se somete a nueva resección de metástasis asociada a RFA, en total 5 lesiones. Además, linfadenectomía extensa iliofemoral izquierda. Biopsia positiva para adenocarcinoma con bordes libres en hígado resecado (RFA no proporciona espécimen) y ganglios con reacción inflamatoria no neoplásica (16 ganglios). Se hospitaliza por 10 días y evoluciona sin complicaciones. Imágenes postoperatorias revelan desaparición de las lesiones (Figura 6).

\section{Caso 4}

Hombre de 57 años, paciente del hospital público, adenocarcinoma colorrectal diagnosticado en abril de 2009, con metástasis hepáticas sincrónicas en hígado derecho más segmento IV. Sometido a neoadyuvancia con esquema FOLFOX. CEA preoperatorio de $420 \mathrm{ng} / \mathrm{ml}$. Se somete a cirugía con intención resectiva del primario, lo que no es posible por sospecha de infiltración perirrectal. Se realiza ligadura portal derecha y se somete a radioterapia rectal abreviada por 5 semanas. A las 8 semanas de ligadura portal, se somete a resección exitosa del primario, sin evidencia anatomopatológica de diseminación radial, y a hepatectomía derecha extendida con bordes negativos (Figura 5).

\section{Caso 5}

Paciente de 60 años, sometido a colectomía parcial en el 2007 por adenocarcinoma. Presenta gran metástasis en segmentos IV y VIII el año 2008, sometiéndose a hepatectomía central tras neoadyuvancia con esquema FOLFIRI. A 24 meses del diagnóstico, presenta recurrencia derecha múltiple y metástasis de $5 \mathrm{~cm}$ en relación con vena suprahepática izquierda.

Se somete a laparotomía exploradora y ligadura portal derecha. Se intenta resecar lesión izquierda, pero se decide preservar la única vena suprahepática en futuro remanente (vena suprahepática media fue resecada previamente en hepatectomía central y derecha saldrá con hepatectomía derecha). A las 8 semanas, la lesión ha crecido y se decide administrar más quimioterapia más bevacizumab. Lesión izquierda reduce a $3,5 \mathrm{~cm}$. Se pesquisa además foco único en colon transverso. Se somete a ablación de metástasis bilaterales y colectomía parcial. Biopsia informa adenocarcinoma con bordes negativos en 
aquello resecado (RFA no proporciona espécimen) Evolución satisfactoria, sin evidencia de enfermedad residual a las imágenes (Figura 7).

\section{Caso 6}

Sesenta y cinco años, paciente del hospital, sometido a resección de primario en marzo de 2009.
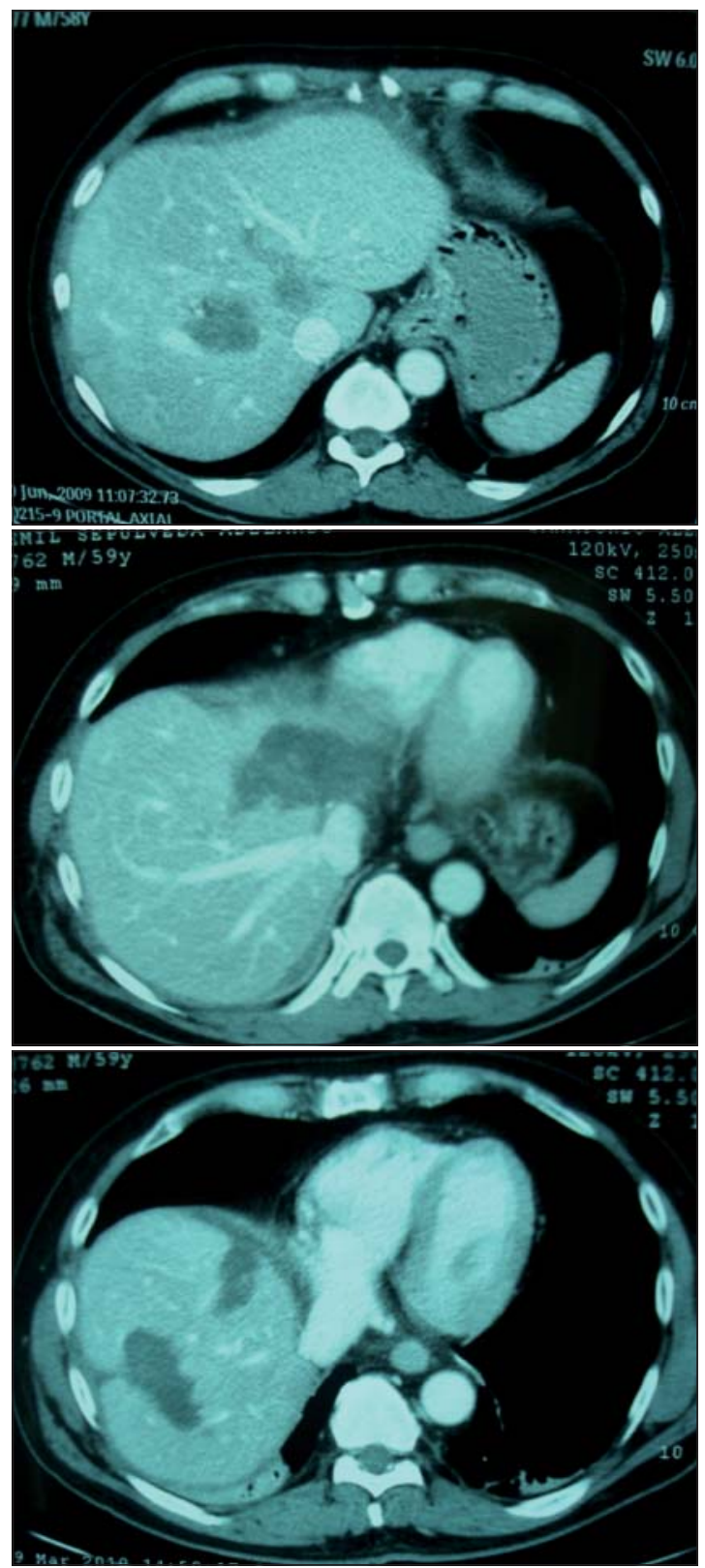

Figura 6. Fotografía superior muestra metástasis que compromete la vena suprahepática izquierda en paciente con hepatectomía central previa. Las dos fotografías inferiores muestran control post cirugía con radiofrecuencia.
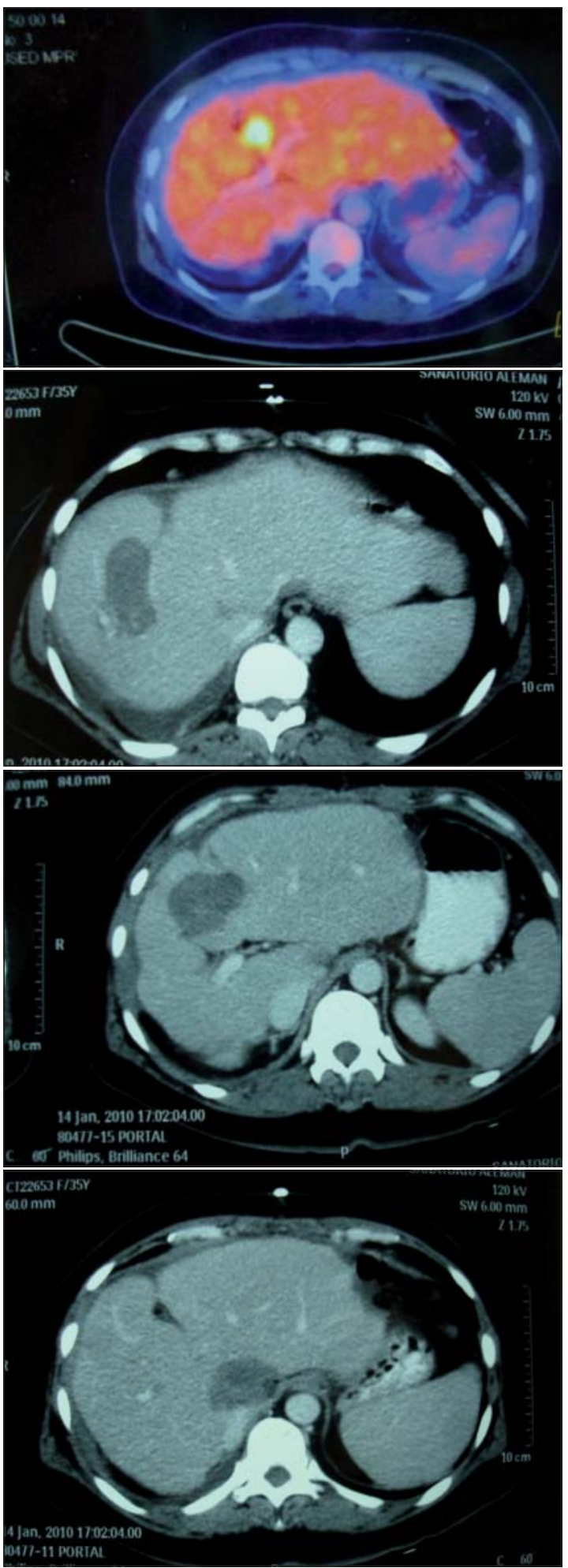

Figura 7. Pet CT muestra una de las lesiones recurrentes en imagen superior. Imágenes inferiores de control post cirugía más radiofrecuencia. 
Se somete a cirugía de revisión de colostomía, con hospitalización prolongada. Recibe neoadyuvancia con esquema FOLFOX. Por metástasis bilaterales se somete a ligadura portal derecha y resección de metástasis de futuro remanente hepático. Cirugía satisfactoria con buena evolución, a pesar de tratarse de un paciente con desnutrición mixta con respuesta discreta a aporte nutricional. Sin evidencia de enfermedad a las imágenes y sin posibilidad de realizarse PET CT. Se somete a hepatectomía derecha extendida a las 8 semanas. Biopsia informa adenocarcinoma con bordes negativos y alejados de resección. Evoluciona con fístula intestinal evidenciada a los 15 días de postoperado. Se reopera evolucionando en forma satisfactoria, aún hospitalizado al momento de este reporte.

\section{Caso 7}

Paciente de 61 años, sometido a hemicolectomía izquierda en hospital de la zona en noviembre de 2009. Evoluciona sin complicaciones. Biopsia revela adenocarcinoma con bordes libres. Se realiza linfadenectomía intercavoaórtica y ligadura portal derecha. Evoluciona sin complicaciones, hospitalizado por 8 días. En espera de hepatectomía de segunda etapa.

\section{Caso 8}

Paciente procedente de Puerto Montt, 55 años. Sometido a colectomía total en ciudad de origen por adenocarcinoma colorrectal. Evoluciona sin complicaciones. Se pesquisa metástasis hepáticas 6 meses post cirugía del primario. Sometido a quimioterapia con buena respuesta. Se somete a resección de enfermedad izquierda y ligadura portal derecha. Evoluciona sin complicaciones, hospitalizado por 12 días. En espera de hepatectomía de segunda etapa.

\section{Discusión}

Esta serie constituye nuestra experiencia inicial con pacientes sometidos a múltiples cirugías en enfermedad hepática metastásica colorrectal bilateral: Resección de primario, hepatectomía en dos etapas y cirugía hepática en 2 etapas con ligadura portal. Considerando que el segmento IV corresponde a hígado izquierdo, el compromiso de hígado derecho y segmento IV también corresponde a enfermedad bilateral aunque no sea parte del futuro hígado remanente.

En general, este tipo de pacientes se asocia a un mal pronóstico ${ }^{1-4}$. La expectativa de sobrevida puede mejorar si transformamos la enfermedad irresecable en resecable. Esto puede lograrse en este subgrupo de pacientes a través de una aproximación multidisciplinaria y de cirugía multietapas ${ }^{1,2,12,13}$.
Alentados por la experiencia internacional y por nuestra experiencia local inicial, pensamos que la hepatectomía en dos etapas es una estrategia factible de realizar para lograr resección curativa en pacientes seleccionados. De todas formas, se trata de pacientes de alto riesgo oncológico y también perioperatorio (resecciones mayores, múltiples cirugías previas, sometidos a quimioterapia y/o radioterapia, etc). Si bien es una serie de tamaño reducido, en todos se logró márgenes negativos. De aquellos que han recurrido, en dos se ha realizado cirugía adicional con resultados satisfactorios. Es importante recordar que en algunas series, la sobrevida mejora respecto de aquellos no resecados, aunque con alta tasa de recurrencia y con pacientes sometidos varias veces a cirugía además de la resección del primario $^{12,13}$.

Se requiere un mayor número de pacientes y un seguimiento a largo plazo para validar este enfoque de manejo en nuestro medio y establecer su papel dentro del tratamiento de las metástasis hepáticas del cáncer colorrectal. Existe experiencia nacional publicada con resultados comparables a la literatura médica internacional ${ }^{14}$. Sin embargo, esta serie es la primera que aborda el manejo multietapas de la enfermedad metastásica hepática bilateral de primario colorrectal en nuestro medio.

\section{Referencias}

1. Adam R, Laurent A, Azoulay D, Castaing D, Bismuth H. Two Stage Hepatectomy: A Planned Strategy to Treat Irresectable Liver Tumors. Annals of Surgery 2000; 232: 777-785.

2. Wicherts DA, Miller R, de Haas RJ, Bitsakou G, Vibert E, Veilhan L, et al. Long-Term results of Two-Stage Hepatectomy for Irresectable Colorectal cancer liver Metastases. Annals of Surgery 2008; 248: 994-1005.

3. Homayounfar K, Liersch T, Schuetze G, Niessner M, Goralczyk A, Meller J, et al. Two-Stage Hepatectomy (R0) with portal vein ligation-towards curing patients with extended bilobular colorectal liver metastases. Int J Colorectal Dis 2009; 24: 409-418.

4. Togo S, Nagano Y, Masui H, Tanaka K, Miura Y, Morioka D, et al. Two-Stage Hepatectomy for Multiple Bilobular Liver Metastases from Colorectal Cancer. Hepato-Gastroenterology 2005; 52: 913-919.

5. Yan T, Sim J, Black D, Niu R, Morris D. Sistematic Review on Safety and Efficacy of Repeat Hepatectomy for Recurrent Liver Metastases from Colorectal Carcinoma. Annals of Surgical Oncology 2007; 14: 2069-2077.

6. Simmonds PC, Primrose JN, Colquitt JL, Garden OJ, Poston GJ, Rees M. Surgical resection of hepatic metastases from colorectal cancer: A systematic review of published studies. British Journal of Cancer 2006; 94 : 982-989. 
7. Jaeck D, Oussoultzoglou E, Rosso E, Greget M, Weber J, Bachellier P. A Two-Stage Hepatectomy Procedure Combined with Portal Vein Embolization to Achieve Curative resection for Initially Unresectable Multiple and Bilobar Colorectal Liver Metastases. Annals of Surgery 2004; 240: 1037-1051.

8. Fong Y, Fortner J, Sun R, Brennan M, Blumgart L. Clinical Score for Predicting Recurrence After Hepatic Resection for metastatic Colorectal Cancer: Analysis of 1001 Consecutive Cases. Annals of Surgery 1999; 230: 309-321.

9. Adam R, Wicherts DA, de Haas RJ, Ciacio O, Lévi F, Paule B, et al. Patients with Initially Unresectable Colorectal Liver Metastases: Is There a Possibility of Cure? Journal of Clinical Oncology 2009; 27: 1829-1835.

10. Pamecha V, Glantzounis G, Davies N, Fusai G, Sharma D, Davidson B. Long-Term Survival and Disease Recurrence Following Portal Vein Embolisation Prior to Major Hepatectomy for Colorectal Metastases. Ann
Surg Oncol 2009; 16: 1202-1207.

11. Mueller L, Hillert C, Möller L, Krupski-Berdien G, Rogiers X, Broering DC. Major Hepatectomy for Colorectal Metastases: Is Preoperative Portal Occlusion an Oncological Risk Factor? Annals of Surgical Oncology 2008; 15: 1908-1917.

12. De Haas RJ, Wicherts DA, Flores E, Azoulay D, Castaing D, Adam R. R1 Resection by Necessity for Colorrectal Liver Metastases: Is it Still a Contraindication to Surgery? Annals of Surgery 2008; 248: 626-637.

13. Adam R, Pascal G, Castaing D, Azoulay D, Delvart V, Paule B, et al. Tumor progression while on chemotherapy: a contraindication to liver resection for multiple colorectal metastases? Annals of Surgery 2004; 240: 1052-1061; discussion 1061-1064.

14. Norero E, Jarufe N, Butte J, Norero B, Duarte I, Torres J, y cols. Resultados del Tratamiento Quirúrgico de Metástasis Hepáticas por Cáncer Colorrectal. Rev Med Chile 2009; 137: 487-496. 